\title{
Inelastic light scattering in magnetic dots and wires
}

\author{
Sergey O. Demokritov and Burkard Hillebrands \\ Fachbereich Physik und Schwerpunkt Materialwissenschaften, Universität Kaiserslautern, 67653 Kaiserslautern, Germany
}

\begin{abstract}
An overview of the current status of the study of spin wave excitations in arrays of magnetic dots and wires is given. We describe both the status of theory and recent inelastic light scattering experiments addressing the three most important issues: the modification of magnetic properties by patterning due to shape anisotropies, anisotropic coupling between magnetic islands, and the quantization of spin waves due to the inplane confinement of spin waves in islands.
\end{abstract}

\section{Introduction}

Progress made during the last decade in lithographic techniques and the analysis of matter enables the fabrication of well-controlled, laterally defined magnetic structures from micron to nanometer sizes. These structures, which usually consist of arrays of separate magnetic islands, are attracting increasing interest due to both fundamental and technological reasons. From the point of view of fundamental studies, both the size and the arrangement of these magnetic islands affect their magnetic properties to a large extend. The reduction of the dimensionality causes new physical phenomena to appear concerning, for example, the magnetization reversal in an island, compared to an infinite film [1]. The high potential for applications of patterned magnetic structures in high-density, low-noise magnetic storage media, magnetic sensors and in magnetic random access memory is obvious [2].

Although static properties of patterned magnetic structures have been studied to some extend $[1,3,4,5]$, highfrequency dynamic properties have been rarely investigated. As the fundamental excitations, we discuss spin waves in the islands. From spin wave measurements basic informations on the magnetic properties, such as magnetic anisotropy contributions, the homogeneity of the internal field, as well as coupling between magnetic islands can be extracted, which are often hard to obtain with other methods. In addition, dynamic excitations define the time scale of a magnetization reversal process, and, therefore, they are of fundamental importance to achieve an understanding of the time structure of the reversal. When the island size becomes comparable to the wavelength of a spin wave under investigation, quantization (or confinement) effects appear, which lead to dramatic changes of the spin wave spectrum and the spin-wave density of states.

\section{Preparation of patterned magnetic structures}

Lateral magnetic structures are conveniently fabricated from magnetic films using lithographic patterning procedures. In the following, for simplicity, we will call structures with one restricted lateral dimension "wires", and those with two restricted dimensions "dots" following the usual conventions, although, as will be one of the subjects of this contribution, no real reduction of dimensionality is given since both in "wires" and "dots" the magnetization is not constant over each magnetic object along the directions of restricted dimension.

Metallic Fe [6], FeNi [7, 8, 9] or Co [10, 11] films are mostly used. For fundamental studies FeNi films are often used due to the smallness of the coercive field and of the intrinsic magnetocrystalline anisotropy. Using this material the field needed to saturate the sample is small, and the vanishing intrinsic anisotropy does not inhibit the observation of sometimes minute anisotropy effects caused by, e.g., the shape of the islands or by interactions between islands. On the other hand, patterned structures made on the basis of Co films allow to investigate the interesting case of perpendicularly magnetized dots or wires [10].

The patterning process is most often performed by means of electron beam lithography (EBL), X-ray lithography (XRL) and by laser interference lithography (LIL), followed by ion beam etching for pattern transfer. Each method has its characteristic advantage. High quality samples can be fabricated using all three processes. EBL is very versatile but due to its serial character it is time consuming, in particular for large pattern areas. XRL is a fast and convenient method, but the need of a synchrotron source and a mask fabrication step hinge its applicability. LIL is a fast and easy process well suited for generating periodic arrays of wires or ellipsoidally shaped islands on large areas with high coherency. Here the magnetic film coated with a photoresist is exposed to the interference pattern produced by two laser beams. Double exposure with a rotation of the sample of $90^{\circ}$ or $120^{\circ}$ generates patterns with fourfold or hexagonal symmetry.

\section{Spin wave in restricted geometries}

The concept of spin waves and their quanta, the magnons, as the lowest lying magnetic states above the ground state was introduced by Bloch in 1930 [12]. Early experimental evidence for their existence came from measurements of thermodynamic properties, but the first direct observation was made by ferromagnetic resonance (FMR) [13]. The first observation of spin waves by inelastic light scattering (Brillouin- or Brillouin-Mandelstam light scattering, BLS) was made by Fleury et al. in 1966 [14]. Since then FMR and BLS have developed to major tools for the characterization of magnetic phenomena.

Spin waves with small wavevectors $\left(\mathrm{q}<10^{6} \mathrm{~cm}^{-1}\right)$, as accessible in a BLS experiment, considered in relatively small propagation distances $(\mathrm{r}<10 \mathrm{~cm})$, are called magnetostatic waves, because the effects of the exchange and retarding 
electro-magnetic fields can be neglected. In restricted geometries spin waves are modified due to the boundary conditions imposed on the components of the magnetic field and the magnetization over the entire surface of the magnetic object. In case of confinement spin waves are no more plain waves, and the assignment of a wavevector to the modes must be made with care. The most simple case, which can be solved analytically for the lowest lying mode, is an ellipsoidally shaped island. Here the demagnetizing field is constant over the island, and a homogeneous distribution of the static part of the magnetization may be assumed. For the lowest lying mode, the distribution of the dynamic components of the magnetization is homogeneous across the island, and the frequency $v$ is determined by the Kittel formula [15]:

$$
v=\frac{\gamma}{2 \pi}\left[\left(H_{i}+N_{x} 4 \pi M_{s}\right)\left(H_{i}+N_{y} 4 \pi M_{s}\right)\right]^{1 / 2}
$$

with $\gamma$ the gyromagnetic ratio and $M_{\mathrm{S}}$ the saturation magnetization aligned along the $\mathrm{z}$-direction. $H_{i}$ is the static internal field defined by

$$
H_{i}=H-N_{z} \cdot 4 \pi M_{s}
$$

and $N_{\mathrm{x}}, N_{y}$ and $N_{z}$ are the demagnetization factors of the ellipsoid.

For the case of an axially magnetized spheroid Walker [16] found a quantization of the magnetostatic modes, which show an inhomogeneous distribution of the dynamic components of the magnetization. These modes, called Walker modes, are characterized by three quantum numbers. For the more general case, e.g., a tangentially magnetized spheroid, no analytic solutions exist to our knowledge for the higher order modes.

To discuss the excitation spectrum in a laterally restricted geometry let us start with the situation of an infinite magnetic film. Here so-called dipolar, magnetostatic surface modes (Damon-Eshbach (DE) modes) exist, if the external field and the magnetization are parallel to the film plane [17]. This mode is a surface mode and it is characterized by an in-plane wave vector $\vec{q}_{\|}$. The mode energy is localized near the film surface and the precession amplitude decays perpendicular to the film with a decay length of the order of $2 \pi / q_{\|}$, which is in the range of $300 \mathrm{~nm}$ in a BLSexperiment. The mode travels parallel to the layers in an angular range close to perpendicular to the applied field in a defined sense of revolution about the film. Neglecting the exchange contribution, the mode frequency for propagation perpendicular to the applied field is:

$$
v=\frac{\gamma}{2 \pi} \cdot\left[H_{i} \cdot\left(H_{i}+4 \pi M_{s}\right)+\left(2 \pi M_{S}\right)^{2} \cdot\left(1-e^{-2 q_{\|} d}\right)\right]^{1 / 2}
$$

with $d$ the film thickness. The internal field $H_{\mathrm{i}}$ is defined here as

$$
H_{i}=H+\beta H_{a}-N \cdot 4 \pi M_{s}
$$

with $H_{a}$ the in-plane anisotropy field $\left(H_{a} \ll 4 \pi M_{S}\right)$. The factor $\beta$ depends on the orientation of the applied field relative to the in-plane easy axis: $\beta=1$, if the field is applied along the easy axis and $\beta=-1$, if the field is applied along the hard axis. Eqs. (1-4) assume a saturation of the magnetization of the sample. The experimental geometry, in which $\vec{q}_{\|} \perp \vec{H}_{i}$, and both vectors are in plane, is called the Damon-Eshbach geometry. The first direct observation of a DE-mode using BLS was made in 1977 by Grünberg and Metawe [18].

For larger film thicknesses so-called (perpendicular) standing spin waves exist, which are of exchange type and which consist of two counter propagating modes traveling almost perpendicular to the films with a wavevector $q=n \pi / d$, with $n$ a positive integer.

For a patterned film the translational symmetry is now broken, and one should expected a geometrical quantization of the in-plane wave vector, $\vec{q}_{\|}$. In the case of wire arrays this quantization should take place, when $\vec{q}_{\|}$is perpendicular to the wire. A calculation of the magnetostatic surface modes in an axially magnetized elliptical cylinder, has been performed by De Wames and Wolfram [19]. They showed, that all modes can be characterized by a positive integer and the wavevector component along the axis of the cylinder, $q_{\mathrm{z}}$. The calculated surface mode frequencies are closely related to the DE-modes of an infinite film. When $q_{\mathrm{z}}=0$, these modes correspond to DE-modes with quantized in-plane wavevector. For $q_{\mathrm{z}} \neq 0$ no simple quantization scheme can be used, because a quantization parameter cannot be defined independent of $q_{\mathrm{z}}$.

In the case of tangentially magnetized dots the problem of spin wave quantization is much more complicated, because even a circular dot cannot be considered as a limiting case of the Walker-mode scenario. In fact, a thin circular disc is a limiting case of a spheroid, when its thickness approaches zero. However, this situation corresponds to a normally, not to a tangentially magnetized disc. To our knowledge there exists up to now no model, which covers this geometry.

\section{Brillouin light scattering}

Two basic techniques, ferromagnetic resonance (FMR) and Brillouin light scattering (BLS), are mainly used for spin wave studies. In the field of patterned structures BLS has a number of advantages over FMR. These are: (i) the potential to investigate spin waves with different absolute values and orientations of their wavevectors; (ii) detection of thermally excited spin waves, i.e., no need to excite spin waves with suitably high wavevectors; (iii) high spatial resolution defined by the size of the laser beam focus, which $30-50 \mu \mathrm{m}$

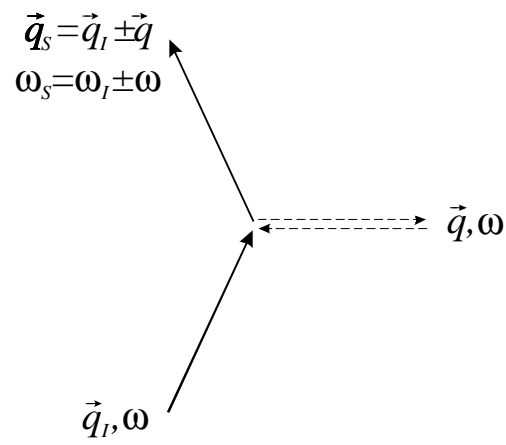

Fig. 1: Scattering process of photons from spin wave excitations. 
in diameter. The latter circumstance allows to investigate small pattern areas, which simplifies the patterning procedure.

Brillouin light scattering is a spectroscopic method for investigating inelastic excitations with frequencies in the $\mathrm{GHz}$ regime. As illustrated in Fig. 1 photons of energy $\hbar \omega_{I}$ and momentum $\hbar \vec{q}_{I}$ interact with the elementary quanta of spin waves $(\hbar \omega, \hbar \vec{q})$, which are the magnons. The scattered photon gains an increase in energy and momentum:

$$
\begin{aligned}
& \hbar \omega_{S}=\hbar\left(\omega_{I}+\omega\right) \\
& \hbar \vec{q}_{S}=\hbar\left(\vec{q}_{I}+\vec{q}\right)
\end{aligned}
$$

if a magnon is annihilated. From Eq. 5 it is evident, that the wavevector $\vec{q}_{S}-\vec{q}_{I}$, transferred in the scattering process, is equal to the wavevector $\vec{q}$ of the spinwave. A magnon can also be created by an energy and momentum transfer from the photon, which in the scattered state has the energy $\hbar\left(\omega_{I}-\omega\right)$ and momentum $\hbar\left(\vec{q}_{I}-\vec{q}\right)$. For finite temperatures $\left(T>>\hbar / k_{B} \approx 1 K\right)$ both processes have about the same probability. In a classical treatment the scattering process can be understood as follows for many materials: Due to magneto-optical effects a phase grating is created in the material, which propagates with the phase velocity of the spin wave. Light is Bragg-reflected from the phase grating with its frequency Doppler-shifted by the spin wave frequency.

The differential light scattering cross section $d^{2} \sigma / d \Omega d \omega_{S}$, i.e., the number of photons scattered into the solid angle $d \Omega$ in the frequency interval between $\omega_{S}$ and $\omega_{S}+d \omega_{S}$ per unit incident flux density, can be written as follows [20]:

$$
\frac{d^{2} \sigma}{d \Omega d \omega_{S}} \propto\left\langle\delta \varepsilon^{*}\left(\vec{q}_{I}-\vec{q}_{S}\right) \delta \varepsilon\left(\vec{q}_{I}-\vec{q}_{S}\right)\right\rangle_{\omega_{I}-\omega_{S}}
$$

with $\delta \varepsilon$ the dynamic (fluctuating) term of the permittivity, which is caused by the spin waves due to magneto-optical effects and which gives rise to the scattering, $\delta \varepsilon$ is proportional to the dynamic part of the magnetization $m$ of the spin wave. The correlation function is given by:

$$
\begin{aligned}
&\left\langle\delta \varepsilon^{*}(\vec{q}) \delta \varepsilon(\vec{q})\right\rangle_{\omega}=\int d\left(t_{2}-t_{1}\right) d^{3}\left(\vec{r}_{2}-\vec{r}_{1}\right) e^{\left[-i \omega t-i \vec{q}\left(\vec{r}_{2}-\vec{r}_{1}\right)\right]} \\
& \times\left\langle\delta \varepsilon^{*}\left(\vec{r}_{1}, t_{1}\right) \delta \varepsilon\left(\vec{r}_{2}, t_{2}\right)\right\rangle \\
& \propto \int d\left(t_{2}-t_{1}\right) d^{3}\left(\vec{r}_{2}-\vec{r}_{1}\right) e^{\left[-i \omega t-i \vec{q}\left(\vec{r}_{2}-\vec{r}_{1}\right)\right]}\left\langle m^{*}\left(\vec{r}_{1}, t_{1}\right) m\left(\vec{r}_{2}, t_{2}\right)\right\rangle
\end{aligned}
$$

with $<\ldots>$ the statistical average. If light is scattered from a spin wave propagating in an infinite medium, the spatial integration volume is the entire space. In this case the correlation function in Eq. (6) is nonzero only if the relations $\omega=\omega_{S}-\omega_{I}$ and $\vec{q}=\vec{q}_{S}-\vec{q}_{I}$ are fulfilled. In this way we yield conservation laws of energy and momentum, described by Eq. (5). However, since for a spin wave mode propagating in a film the integration volume is bounded by the two film surfaces, the conservation conditions are fulfilled only

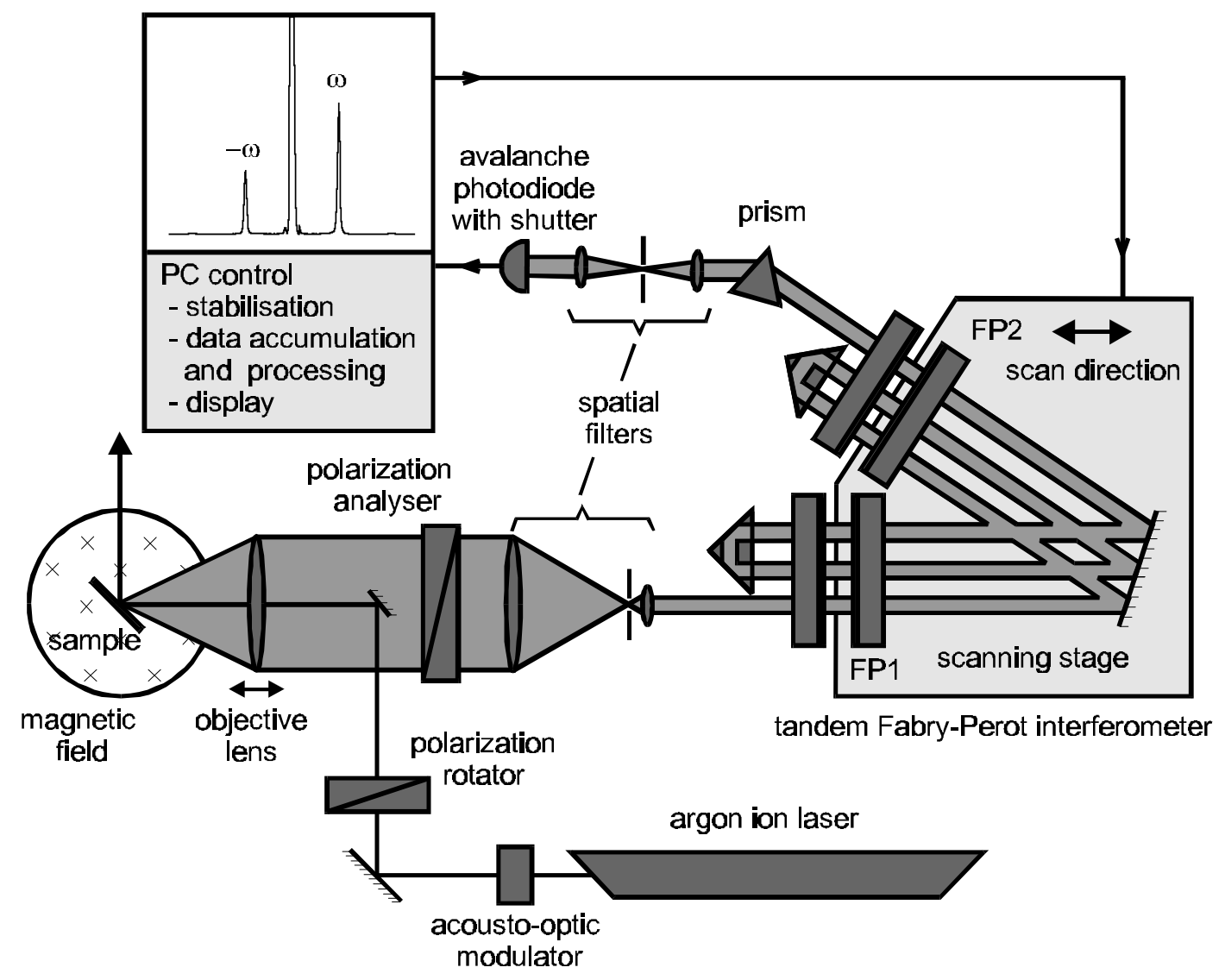

Fig. 2: Schematic view of a Brillouin light scattering setup. 
for the two in-plane components of the wavevector, $\vec{q}_{\|}$. In backscattering geometry, when $\vec{q}_{S}=-\vec{q}_{I}, \vec{q}_{\|}$is determined by the angle of incidence of the light, $\theta: q_{\|}=2 q_{I} \sin \theta$. The third component perpendicular to the film, $q_{n}$, is not well defined by the conservation law because the system does not possess the symmetry of translational invariance perpendicular to the film. The uncertainty in $q_{n}$ is, apparently, inversely proportional to $t$, the thickness of the film or of the mode localization region. It is negligible, if $\left(\vec{q}_{S}-\vec{q}_{I}\right)_{n} t>>2 \pi$.

If now the in-plane translational invariance of the magnetic film is broken by patterning, the in-plane wavevector, $\vec{q}_{\|}$, is not anymore fully conserved in a the light scattering process. In the case of a spin wave mode localized in a wire, for example, the only conserved component is the component of $\vec{q}_{\|}$along the wire axis. It is clear from Eq. (7), that the dependence of the differential light scattering cross section on the component of $\vec{q}_{\|}$perpendicular to the wires is determined by the Fourier component of $m\left(\vec{r}_{\|}\right)$. We will discuss this in more detail in Sect. 5.

A typical experimental setup for BLS studies is schematically shown in Fig. 2. Light of a frequency stabilized laser $(\Delta v=20 \mathrm{MHz})$, which is typically an $\operatorname{Argon}^{+}$-ion laser $(\lambda=$ $514.5 \mathrm{~nm}$ ), is focused onto the sample with an objective lens. The light scattered from the sample (elastic and inelastic contributions) is collected and sent through a spatial filter for suppressing background noise before entering the tandem interferometer. The central part of the interferometer consists of two Fabry-Perot etalons FP1 and FP2. The tandem arrangement avoids ambiguities in the assignment of inelastic peaks to the corresponding transmission order $[21,22]$. In order to obtain the high contrast necessary to detect the weak inelastic signals, the light is sent through both etalons several times using a system of retroreflectors and mirrors. The frequency selected light transmitted by the interferometer is detected by a photomultiplier or an avalanche photodiode after passing through a second

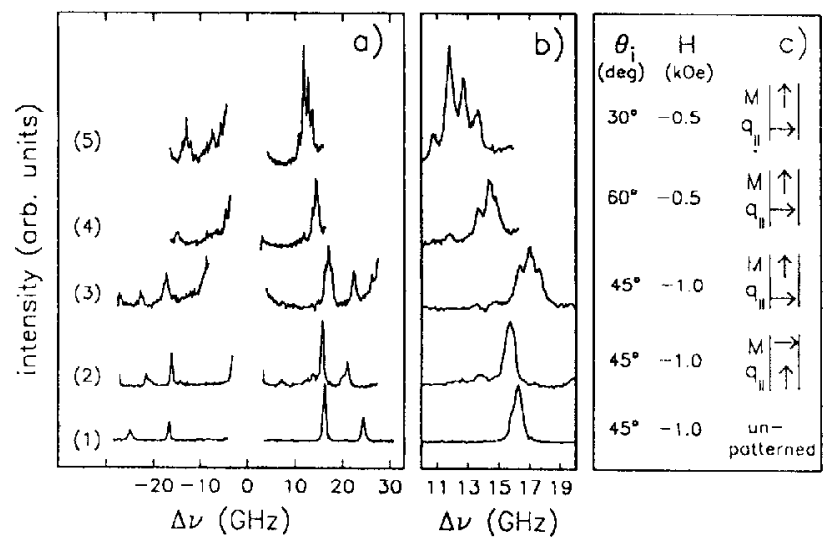

Fig. 3: a) BLS spectra of the NiFe wire array (2-5) compared to the spectrum of the unpatterned film (1); b) shown is the region of interest from 10 to $20 \mathrm{GHz}$ in detail; c) diagrams indicating the angle of incidence $\theta_{i}$, the applied field $H$, and the experimental geometry (from [23]). spatial filter for additional background suppression. A prism or an interference filter between the second spatial filter and the detector serves for suppression of inelastic light from common transmission orders outside the frequency region of interest. Data collection is performed by a personal computer or by a multichannel analyzer.

\section{Arrays of wires}

The spin wave excitation spectrum of arrays of ferromagnetic wires was recently investigated by several groups $[5,9$, 11, 23, 24, 25].

An early investigation of BLS from spin waves in an array of magnetic wires made of permalloy was performed by Gurney et al. [23]. The wire thickness and width were 30 $\mathrm{nm}$ and $0.55 \mu \mathrm{m}$, respectively. As it is shown in Fig. 3, a splitting of the spin wave spectrum into several discrete modes was observed in Damon-Esbach mode geometry at a few values of the in-plane wavevector $q_{\|}$. The authors were not able to identify the nature of the modes.

Ercole et al. [9] investigated arrays of wires, fabricated from $60 \mathrm{~nm}$ thick, Au captured FeNi films grown on GaAs(001) substrates. Wire arrays were produced with wire widths between 0.4 and $10 \mu \mathrm{m}$, the individual wires were separated by twice their width. The surface Damon-Eshbach spin wave modes and the lowest-lying volume modes were observed. The spin wave frequencies were found to be sensitive to the size of the wires. The measured spin wave frequencies as a function of the in-plane angle of the wavevector of the spin waves demonstrate a clear twofold symmetry, obviously introduced by the symmetry of the wire orientation. Both effects have a magneto-dipole origin and are due to demagnetization fields caused by the wire edges. The magnetic easy axis corresponds to the case when the wires are parallel to the applied field. No evidence of any mode splitting was reported. The authors have reproduced numerically their experimental results by solving the Landau-Lifshitz torque equation with the dynamic magnetization profiles assumed to be homogeneous in each wire. As a result, an expression for the spin wave frequency was obtained, which is close to the Kittel-formula Eq. (1), describing the $q=0$ resonance in finite samples.

Cherif et al. [11] studied spin waves in arrays of $40 \mathrm{~nm}$ thick Co wires with widths and periodicities on the micrometer scale. Contrary to $\mathrm{FeNi}$, Co films possess an essential magnetic anisotropy with an effective field $\mathrm{H}_{\mathrm{a}}=2.6 \mathrm{kOe}$. Under a magnetic field parallel to the wire, the frequency of the DE-mode is higher than in the unpatterned film. For a magnetic field perpendicular to the wire a significant variation of the frequency is observed, which is related to the size-dependent demagnetizing field. An additional, low frequency mode which is not seen in the infinite film, was observed. The authors connect this mode with the uniform precession mode, which gives rise to a measurable BLS intensity in patterned, highly anisotropic structures.

Mathieu et al. [24] and Jorzick et al. [26] investigated spin wave excitations by BLS in arrays of FeNi wires. In addition to demagnetization effects a quantization of the spin 
wave mode in several dispersionless modes was observed and quantitatively described. This quantization is connected to a confinement effect of the spin waves. It was not seen in the geometry when the wavevector of the detected mode was parallel to the wires. Since these experiments provide the first account for a quantitative understanding of $(q \neq 0)$ spin wave quantization effects in lateral structures, let us consider these experiments in more detail.

The samples were made of a $20 \mathrm{~nm}$ and $40 \mathrm{~nm}$ thick $\mathrm{Ni}_{81} \mathrm{Fe}_{19}$ film deposited in UHV onto a $\mathrm{Si}(111)$ substrate by means of e-beam evaporation. Patterning was performed using X-ray lithography with a following lift-off process with Al coating and ion milling [27]. The procedure is a high quality patterning process, which provides a superb quality of the wire boundaries. Several types of periodic arrays of wires with wire widths $w=1.7 \mu \mathrm{m}$ and $1.8 \mu \mathrm{m}$ and distances between the centers of the wires, $\Lambda$, of 2.5 and $4 \mu \mathrm{m}$ (i.e., wire separations of $0.7,0.8,2.2$ and $2.3 \mu \mathrm{m}$ ) were prepared. The length $L$ of the wires was $500 \mu \mathrm{m}$. The patterned area was $500 \times 500 \mu \mathrm{m}^{2}$. An investigation of the magnetization reversal behavior of the structures, performed by Kerr magnetometry, showed that the magnetic easy axis of the array was along of the wire axis.

In a BLS experiment the in-plane wavevector $\vec{q}_{\|}=\left(\vec{q}_{S}-\vec{q}_{I}\right)_{\|}$, transferred in the light scattering process,

was oriented perpendicular to the wires and its value was varied by changing the angle of light incidence, $\theta$, measured against the surface normal: $q_{\|}=\left(4 \pi / \lambda_{\text {Laser }}\right) \cdot \sin \theta$. The collection angle of the scattered light was chosen small enough to ensure a reasonable resolution in $q_{\|}$of $\pm 0.8 \cdot 10^{4} \mathrm{~cm}^{-1}$. It is important to mentioned here once more, that, strictly speaking, $\vec{q}_{\|}$cannot be considered as the wavevector of the spin waves mode taking part in the scattering process and, thus, tested in the experiment.

Let us at first consider the results of the experiments,. Fig. 4 shows the anti-Stokes side of a typical BLS spectrum of a wire array with a wire thickness $d=20 \mathrm{~nm}$, width $w=1.8$ $\mu \mathrm{m}$, and separation between the wires $\delta=0.7 \mu \mathrm{m}$ for

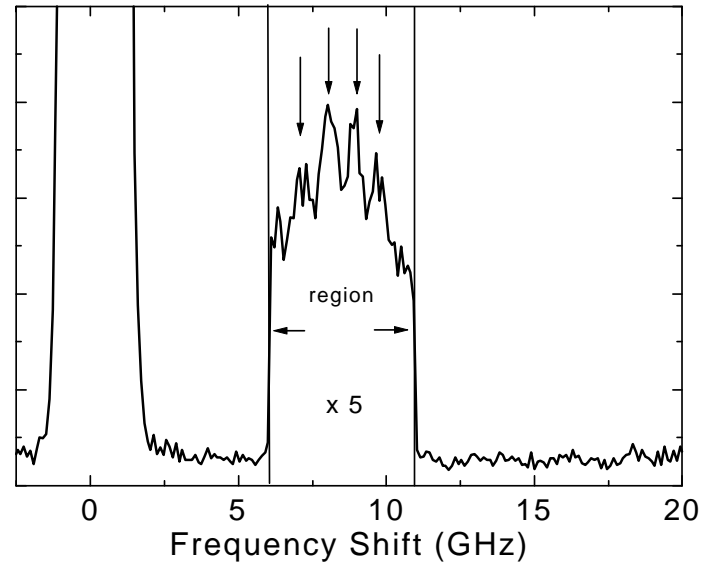

Fig. 4: $\quad$ BLS spectrum of a wire array with a wire width of $1.8 \mu \mathrm{m}$, a wire thickness of $20 \mathrm{~nm}$ and a wire separation of $0.7 \mu \mathrm{m}$, demonstrating the existence of several discrete spin wave modes (indicated by arrows) with a mode splitting of about $0.9 \mathrm{GHz}$. The applied field is 500 Oe. The transferred inplane wavevector $q_{\|}$is $0.3 \cdot 10^{5} \mathrm{~cm}^{-1}$. The intensity at zero frequency shift is due to elastically scattered light. In the region of interest the scan speed was reduced by a factor of five (from [24]). $q_{\|}=3 \cdot 10^{4} \mathrm{~cm}^{-1}$. Near $7.1,8.0,8.8$ and $9.6 \mathrm{GHz}$ four distinct modes of magnetic excitations are observed. Fig. 5 shows both sides of a BLS spectra for thicker wires with $d=40$ $\mathrm{nm}, w=1.7 \mu \mathrm{m}$ and $\delta=0.8 \mu \mathrm{m}$ for the same values of the applied field and the transferred wavevector. Besides a splitting in the DE mode corresponding to in-plane quantized spin wave modes an exchange dominated, perpendicular standing spin wave (PSSW) mode is seen in Fig. 5 near $14 \mathrm{GHz}$. It is not visible in Fig. 4, since its frequency strongly depends on the film thickness $\left(\omega \propto d^{-2}\right)$. The different mode character of the DE modes and the standing spin wave is evidenced by the respective ratio of the antiStokes and Stokes peak intensities.

By varying the magnitude of the wavevector, $q_{\|}$, the spin wave dispersion was obtained for both thicknesses of the wires, as displayed in Fig. 6. Shown are the data for the wires with $\Lambda=4 \mu \mathrm{m}$ (open symbols), and $2.5 \mu \mathrm{m}$ (solid symbols). In the region of low wavevectors the spin wave modes show a disintegration of the continuous dispersion of the Damon-Eshbach mode of an infinite film into several discrete, resonance-like modes with a frequency spacing between the lowest lying modes of approximately $0.9 \mathrm{GHz}$ for $d=20 \mathrm{~nm}$ and $1.5 \mathrm{GHz}$ for $d=40 \mathrm{~nm}$. There is no significant difference between the data obtained from the arrays with $\Lambda=2.5 \mu \mathrm{m}$ and $\Lambda=4 \mu \mathrm{m}$, indicating that the mode splitting is purely caused by the quantization of the spin waves due to the finite wire width, and the magnetodipole interaction between wires is small for both studied wire separations and thicknesses.

The main features observed in Figs. $6 \mathrm{a}$ and $6 \mathrm{~b}$ are as follows: (i) For low wavevector values $\left(\cong 0-1.0 \cdot 10^{5} \mathrm{~cm}^{-1}\right)$ the discrete modes do not show any noticeable dispersion and they behave like standing wave resonances. (ii) The discrete modes are each observed over a continuous range of the transferred wavevector, $q_{\|}$. (iii) The lowest mode appears near zero wavevector $\left(\mid q \| \leq 0.08 \cdot 10^{5} \mathrm{~cm}^{-1}\right)$, whereas all higher modes appear at higher wavevectors, and the value of the respective lower "cut-off" wavevector increases with the mode number. (iv) There is a transition region $\left(q_{\|} \cong 1.0-1.3 \cdot 10^{5} \mathrm{~cm}^{-1}\right)$ between the well resolved dispersionless modes, and the continuous film-like dispersion,

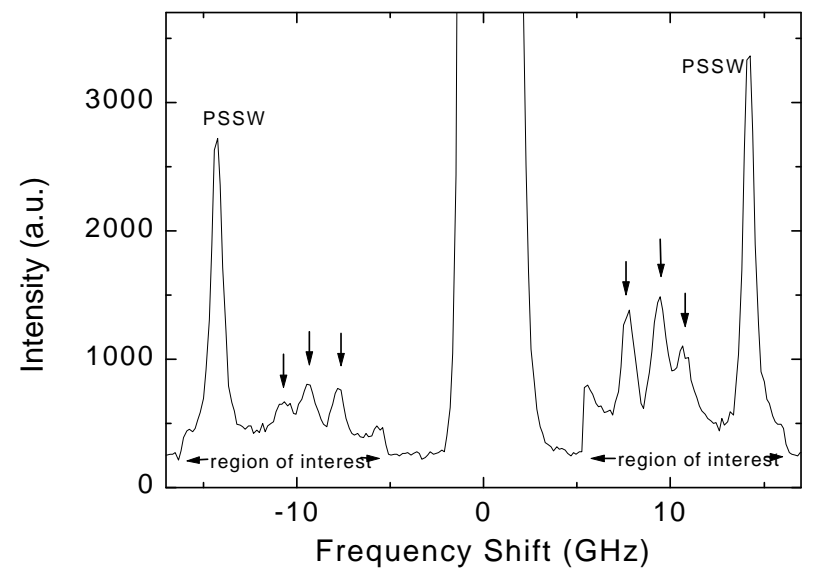

Fig. 5: $\quad$ BLS spectrum of the wire array with a wire width of $1.7 \mu \mathrm{m}$, a wire thickness $40 \mathrm{~nm}$ and a wire separation of $0.8 \mu \mathrm{m}$. The applied field is 500 Oe. The transferred in-plane wavevector $q_{\|}$is $0.3 \cdot 10^{5} \mathrm{~cm}^{-1}$. In the regions of interest $( \pm(5-17) \mathrm{GHz})$ the scan speed was reduced by a factor of three. 
where are discrete modes, but the mode separation is close or slightly below the experimental frequency resolution in the BLS experiment $(\approx 0.1 \mathrm{GHz})$. $\quad(\mathrm{v})$ For large values of the wavevector $\left(q_{\|}>1.3 \cdot 10^{5} \mathrm{~cm}^{-1}\right)$ the dispersion of the patterned film converges to the dispersion of a continuous film.

Of fundamental interest is also the BLS cross section. The interesting problem is, how the BLS cross section depends on $q_{\|}$for each of the quantized modes. In general, it is very difficult to measure absolute values of the scattering cross section or even to compare the intensities of modes between different BLS spectra. However, as it is seen in Fig. 6b, the exchange dominated, perpendicular standing spin wave mode is observed in all the spectra with $d=40 \mathrm{~nm}$ for the entire investigated range of $q_{\|}$. Therefore, it can be used as a reference for relative measurements of the scattering cross section. The intensity of each in-plane mode, normalized to the intensity of the perpendicular standing mode is shown

a)

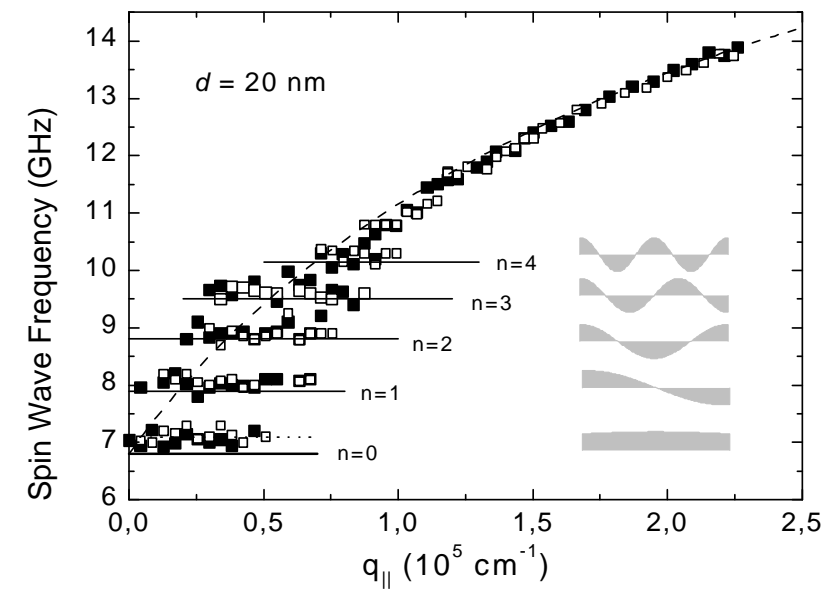

b)

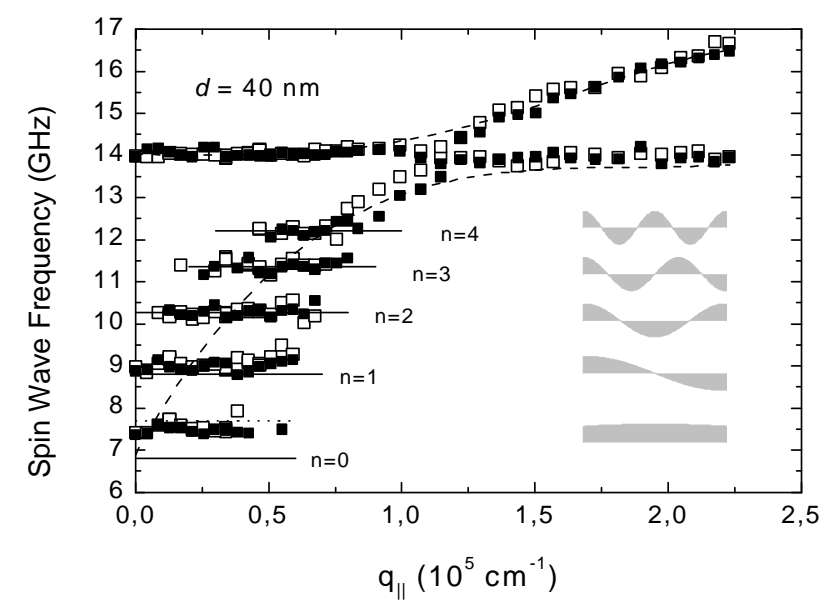

Fig. 6: Obtained spin wave dispersion curves for an array of wires of a periodicity $\Lambda=2.5 \mu \mathrm{m}$ (open squares) and $4 \mu \mathrm{m}$ (solid squares).. The external field applied along the wire axes is 500 Oe. The solid horizontal lines indicate the results of a calculation using Eqs. $(3,8)$, the dotted horizontal line indicates the result of calculations for the modes with $n=0$ using Eqs.(1,2). a) the thickness of the wires is $d=20 \mathrm{~nm}$, the dashed line is the calculated DamonEshbach dispersion of an infinite film using Eq. (3) plotted for reference; b) d $=40 \mathrm{~nm}$, the dashed line indicates the result of the calculated hybridized dispersion of a DE-mode and the first perpendicular standing spin-wave mode in an infinite film for reference. by black squares in Fig. 7 .

In order to understand the above experimental results, the main issues are (i) to calculate the mode frequencies and (ii) to understand why each of the modes is observed over a characteristic continuous range of wavevectors. For the discussion we assume a Cartesian coordinate system oriented such that the normal of the pattern area points into the $x$-direction, the wavevector $\vec{q}_{\|}$points into the $y$ direction, and the wire axes, the applied magnetic field and the magnetization are all aligned parallel to the $z$-axis.

In the case of small values of $q_{\|}$it is natural to assume that the observed discrete spin wave modes result from the width-dependent quantization of the in-plane wavevector $q_{\mathrm{n}}$ in the DE-dispersion equation, described by Eq. (3) with a static demagnetizing field $H_{\mathrm{d}}=0$. This approach is valid since the magnetic field is applied along the wires and the aspect ratio in this case is very high. The quantized values of $q_{\|, \mathrm{n}}$ (or, correspondingly, the quantized wavelength $\lambda_{\mathrm{n}}$ ) in a thin $(d<<w)$ magnetic wire can be obtained by imposing boundary conditions on the variable magnetization $m$ at the side walls of the wire $\partial m /\left.\partial y\right|_{y= \pm w / 2}=0$, corresponding to zero surface anisotropy:

$$
w=n \frac{\lambda_{n}}{2} ; \quad q_{\|, \mathrm{n}} \equiv \frac{2 \pi}{\lambda_{n}}=\frac{\pi}{w} n,
$$

with $n$ the mode index, $n=0,1,2 \ldots$ The mode frequencies are obtained by inserting the discrete values $q_{\|, \mathrm{n}}$ into the Damon-Eshbach dispersion, Eq. (3). The profiles of the dynamic component of the magnetization $m(y)$ across the wire describing the in-plane standing waves are:

$$
m_{n}(y)=a_{n} \cos \left(q_{\|, n}(y+w / 2)\right), \quad-w / 2<y<w / 2 \text {. }
$$

with $a_{\mathrm{n}}$ the corresponding mode amplitudes. This approach should yield reasonable estimates for the frequencies of the quantized modes since the film thickness is much smaller than the width of the wires. For the calculation we use the independently measured values of the material parameters $4 \pi M_{\mathrm{S}}=10.2 \mathrm{kG}, \gamma / 2 \pi=2.95 \mathrm{GHz} / \mathrm{kOe}$. The results of the

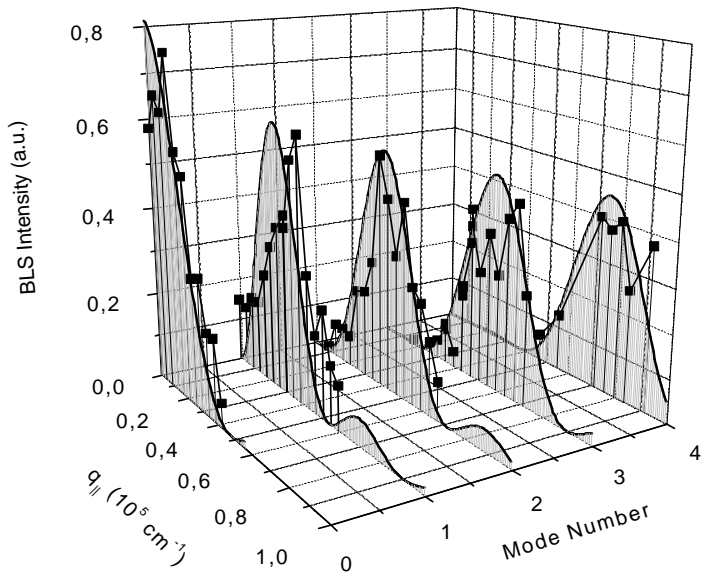

Fig. 7: $\quad$ Measured relative BLS intensities (black squares) of the in-plane quantized spin wave modes for as a function of the wavevector $q_{\|}$and the mode number $\mathrm{n}$ in comparison to the calculated results based on Eqs. (8-10) (gray colored curves). 
calculation are shown in Fig. 6 by solid horizontal lines together with the experimental data. Without any fit parameters the calculation reproduces all mode frequencies with $n>0$ very well, and for the $n=0$ mode a reasonable agreement is achieved. Since the group velocity $V_{\mathrm{g}}=\partial \omega / \partial q$ of the dipolar surface spin wave (cf. Eq. (3)) decreases with increasing wavevector, the frequency splitting of neighboring, width-dependent discrete spin wave modes, which are equally separated in $q$-space $\left(q_{\|, \mathrm{n}}=n \pi / w\right)$, becomes smaller with increasing wavevector $q_{\|, \mathrm{n}}$, until the mode separation is smaller than the frequency resolution in the BLS experiment and/or the natural line width, and the splitting is not anymore observable. Fig. 8 demonstrates the evolution of the mode frequencies with an increasing applied field, showing that the splitting decreases with increasing applied field.

Two aspects were not considered in the above discussion: (i) a possible pinning of the spins at the side walls of the wires was not taken into account. This assumption is justified since anisotropies in permalloy are small. In contrary, when pinning is active, a boundary condition analogous to the Rado-Weertman boundary condition of an infinite film must be imposed at the side walls, which results in a phase shift of the spin waves upon reflection of the side walls [25]; (ii) the dynamic dipole fields in a wire with a non-zero, even though small, ratio $d / w$ differ from those of an infinite film. Both effects slightly change the frequencies of the modes, but with increasing mode number they become more and more negligible. The former effect should not be important in NiFe due to its small anisotropy. The latter effect changes the frequency of the uniform $(n=0)$ precession mode and can be easily calculated by means of the Kittel formula, Eq. (1), using demagnetization factors of the wires, measured independently. The obtained value is shown in Fig. 6 by a dotted line. The agreement with the experiment is excellent.

As it was pointed out above, the light scattering cross section at a given transferred wavevector is determined by the corresponding Fourier component of the spin wave mode

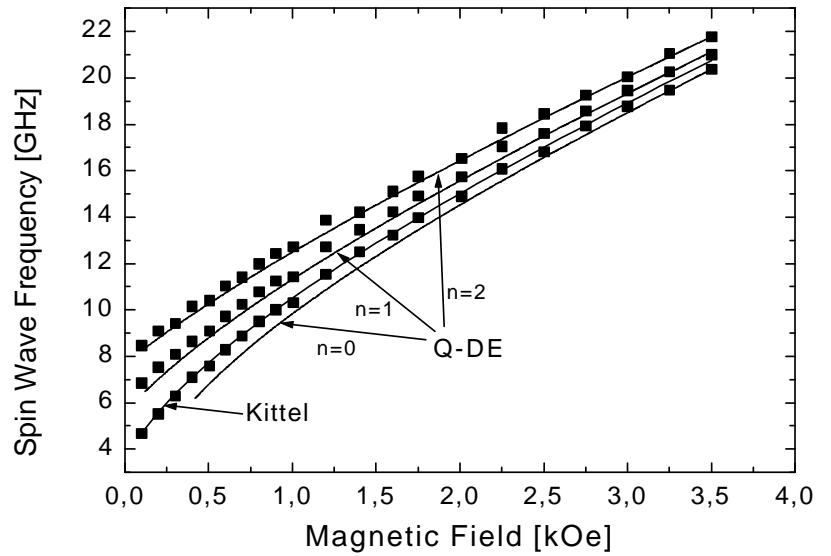

Fig. 8: Frequencies (for $q_{\|}=0.3 \cdot 10^{5} \mathrm{~cm}^{-1}$ ) of the in-plane quantized spin wave modes of the wire array with a wire width of $1.7 \mu \mathrm{m}$, a wire thickness 40 $\mathrm{nm}$ and a wire separation of $0.8 \mu \mathrm{m}$ as a function of the applied field. The lines are calculated using either the DE-equation with quantized wavevectors (indicated by Q-DE) or the Kittel formula (Eq. (1)) as indicated. profile $m(\vec{\eta})$. For a spin wave mode existing in the wire of width $w, m$ is zero outside the wire, and therefore not a periodic function in space. Then the Fourier component $m\left(q_{\|}\right)$is a continuous function of $q_{\|}$given by:

$$
m\left(q_{\|}\right)=\int_{-w / 2}^{w / 2} m(y) \exp \left(i q_{\|} y\right) d y .
$$

Using Eqs. (8-10), the Fourier components $\left|m_{\mathrm{n}}\left(q_{\|}\right)\right|$for the discrete modes are calculated. The resulting cross section profiles $I \propto\left|m_{\mathrm{n}}\left(q_{\|}\right)\right|^{2}$ of the lowest five modes for the arrays with the wire thickness $d=40 \mathrm{~nm}$ are shown in Fig. 7. There is a good agreement between the results of this calculation and the experiment.

\section{Arrays of dots}

The investigation of arrays of magnetic dots is even more challenging then the study of arrays of magnetic wires. Wires always have a shape anisotropy caused by demagnetization fields. It means that spin waves in wires are not degenerated, and possible effects of a weak interaction between the wires cannot easily be seen in a BLS experiment. As additional degrees of freedom one can vary the shape of the dots (circle, ellipse, square,...) as well as their arrangement (rectangular, square, hexagonal lattice). Last but not least, arrays of isolated magnetic dots are a good model system to study fundamental physical aspects of the next generation magnetic random access memory.

Up to now there exist very few studies of magnetic dot arrays by BLS $[6,7,8,28]$. This is definitely connected to the fact that, on one hand, it is much more difficult to prepare dots with a well defined shape; and on the other hand, that the BLS cross section from dots is lower than that from wires simply due to the lower coverage of the surface by the magnetic material.

Grimsditch et al. [6] investigated sub-micron Fe magnetic dot arrays by BLS with dots of ellipsoidal shape. They showed that the shape anisotropy of individual dots is a dominant source of anisotropy, measured both by static magnetometry and BLS. The measured spin wave frequen-

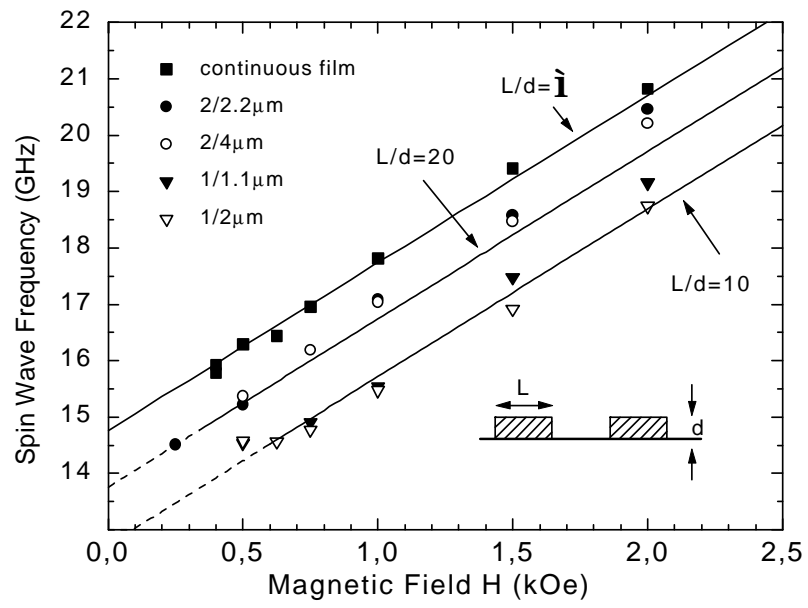

Fig. 9: Measured spin wave frequencies of the dot arrays with a dot thickness of $\mathrm{d}=100 \mathrm{~nm}$ as a function of the magnetic field. The solid lines represent the calculated frequencies on the basis of Eqs. $(3,4)$ using aspect ratios as indicated (from [7]). 
cies are in good agreement with values calculated on the basis of isolated ellipsoids using the Kittel formula, Eq. (1). No inter-dot coupling was observed.

Hillebrands et al. [7, 28] investigated spin wave properties of square lattices of micron-sized dots of permalloy with varying dot separations. Eight different samples comprising circular dots arranged in a $1 \times 1 \mathrm{~mm}^{2}$ square lattice with a diameter/periodicity of $1 / 1.1,1 / 2,2 / 2.2$ and $2 / 4 \mu \mathrm{m}$, respectively, patterned into 500 and $1000 \AA$ thick films were prepared. Special care was taken to avoid a touching of neighboring dots even for the smallest separation. This was confirmed by depth profile measurements. The measured spin wave frequencies of the different dot arrays as a function of the applied field are shown in Fig. 9. For each dot array the spin wave frequencies decrease with decreasing field, and they disappear below certain critical applied fields. The strong reduction of the spin wave frequencies with decreasing aspect ratio is also seen in Fig. 9. This is caused by size depending demagnetizing fields (cf. Eq.(4)), i.e., the demagnetizing factor $N_{\mathrm{z}}$ of each magnetic dot decreases with the aspect ratio. The solid lines in Fig. 9 are calculated on the basis of Eqs. $(3,4)$ using demagnetizing factors of spheroids with axial ratios taken from the aspect ratios of the magnetic dots. Although spheroids are a crude approximation to the real three-dimensional shape of the dots, the calculation is in reasonably good agreement with the experimental data for $H>N_{\mathrm{z}} \cdot 4 \pi M_{s}$.

To investigate the problem of an in-plane inter-dot coupling, the spin wave frequencies were measured as a function of the angle of the in-plane applied field, $\Phi_{\mathrm{H}}$, with respect to a reference [10]-direction of the lattice arrangement. For the smallest dot separations of $0.1 \mu \mathrm{m}$ a fourfold anisotropic behavior was found, which was seen neither for larger dot separations nor for the unpatterned reference film. This is displayed in Fig. 10 for the $1 / 1.1 \mu \mathrm{m}$ and, for comparison, for the $1 / 2 \mu \mathrm{m}$ lattices of the sample of $100 \mathrm{~nm}$ thickness at an applied field of $1 \mathrm{kOe}$. Note here that the

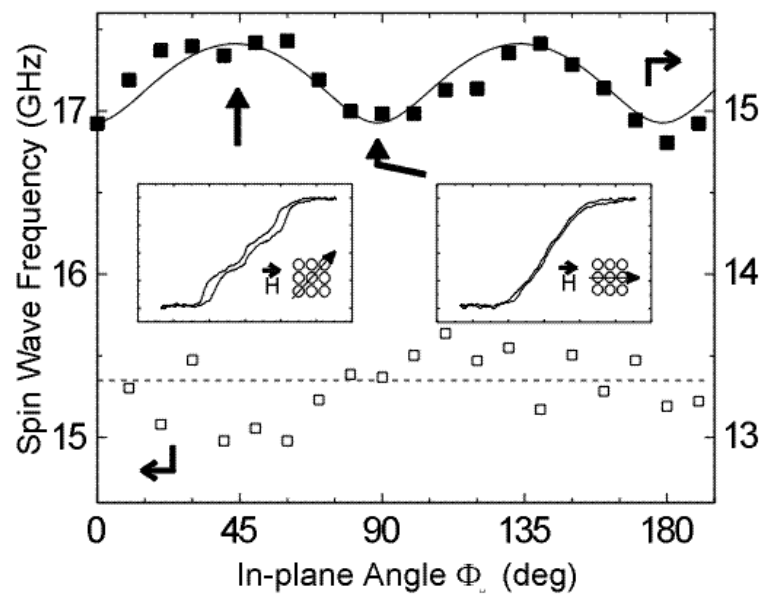

Fig. 10: Dependence of the spin wave frequencies on the in-plane direction of the applied field for the $1 / 1.1 \mu \mathrm{m}$ (full squares) and, for comparison, for the $1 / 2 \mu \mathrm{m}$ (open squares) dot arrays of $100 \mathrm{~nm}$ thickness. The solid line is a fit for the data. Magnetization curves of the $1 / 1.1 \mu \mathrm{m}$ dot lattice are shown as insets with the applied field as indicated. Note that maximum values of the spin-wave frequencies indicate an easy axis (from [28]). easy axes (maximum frequencies) of the observed anisotropy are along the [11]-directions of the lattice. To determine quantitatively the anisotropy constant the free energy expression

$$
F=K^{(4)} \sin ^{2} \Phi \cdot \cos ^{2} \Phi
$$

with $\Phi$ the angle between the direction of magnetization with respect to the [10]-direction, and $\mathrm{K}^{(4)}$ the constant of a fourfold in-plane anisotropy. A model fit using Eq. (11) and a numerical procedure to calculate the spin wave frequencies [29] is displayed in Fig. 10 as a solid line for the $1 / 1.1 \mu \mathrm{m}$ lattice. For both $1 / 1.1 \mu \mathrm{m}$ samples with thicknesses of $50 \mathrm{~nm}$ and $100 \mathrm{~nm}$ the anisotropy contribution $\mathrm{K}^{(4)}$ was determined for several values of the applied magnetic field. The obtained values of $\mathrm{K}^{(4)}$ decrease with increasing field and saturate within the investigated field range at $\mathrm{K}^{(4)}$ $=-0.6 \cdot 10^{5} \mathrm{erg} / \mathrm{cm}^{3}$, which corresponds to an effective anisotropy field $H_{\text {ani }}=150 \mathrm{Oe}$, at the same reduced field value of about $H / H_{\mathrm{d}}=5$ with $H_{\mathrm{d}}$ the demagnetizing field. As a function of the reduced field, $H / H_{\mathrm{d}}$, the data of both dot thicknesses fall onto one common curve within the error margins indicating that the coupling strength scales with the demagnetizing field. The observed fourfold anisotropy can be understood as being caused by a magneto-dipole interaction between residual unsaturated parts of the dots. Because of the large distance of $0.1 \mu \mathrm{m}$ between the dots a direct exchange mechanism via conduction electrons or via electron tunneling can be excluded. A dipolar interaction of completely magnetized dots also cannot account for the observed anisotropy, because the corresponding dipolar energy can be expressed as a bilinear form of the components of the magnetization vector. Such an expression can only yield an uniaxial, but not a fourfold anisotropy contribution, since in a bilinear form the direction cosines appear quadratic in highest order and add to a constant if a fourfold symmetry is given. But, if the dots are not completely saturated, the magneto-dipole interaction energy cannot be expressed in the above bilinear form, and the fourfold anisotropy is not anymore forbidden. The large observed decrease of the coupling anisotropy constant with increasing

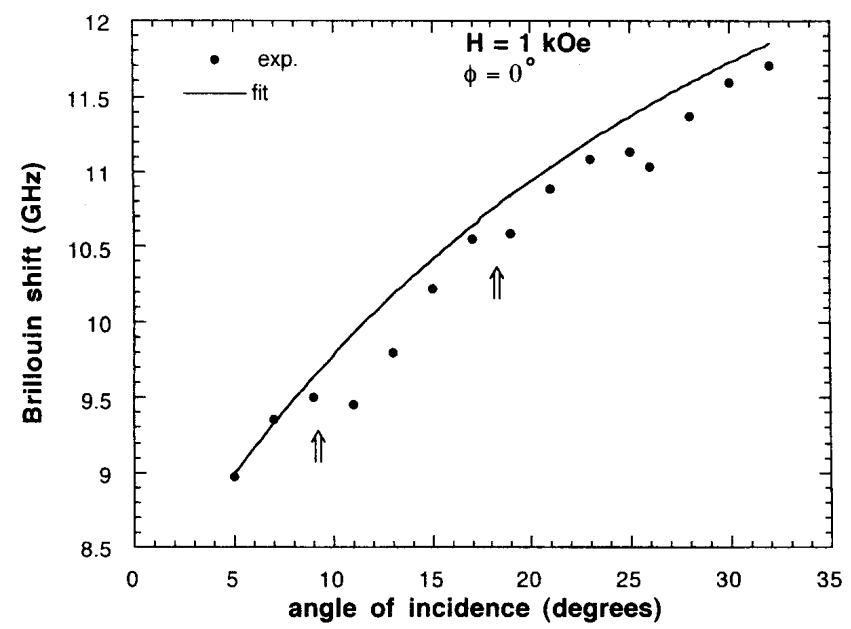

Fig. 11: Dependence of the spin wave frequencies of the arrays of square dots with an edge length of $2 \mu \mathrm{m}$ as a function of the angle of light incidence. The solid line is calculated on the basis of Eq. (3). The arrows indicate the step-like misfits due to possible spin wave quantization (from [8]). 
field, forcing the alignment of the magnetization with the field, corroborates this assumption.

Cherif et al. [8] studied magnetic properties in periodic arrays of square permalloy dots. The spin wave frequencies were found to be sensitive to the size of the dots and, for the smallest structures, to the in-plane direction of the applied field. While the size dependence can be reasonably explained to originate from the demagnetizing field effect, the in-plane anisotropy should be discussed in more detail. The authors observed a fourfold anisotropy in the spin wave frequencies as a function of the in-plane angle of the external field. The spin wave frequencies show maxima for the magnetic field applied along the edges of the squares, indicating easy axes of anisotropy along the edges. Contrary to the results of Hillebrands et al [7, 28], the authors did not demonstrate that the observed anisotropy is caused by interdot interaction, instead they argue that it may be a singledot effect. In fact, dots of square shape possess a lower symmetry compared to circular shaped dots. Therefore, shape affects (corner effects for the magnetization, confinement of the spin wave mode etc.) can cause this anisotropy. An indication of one of these effects, obtained by Cherif et al., is shown in Fig. 11. the figure shows the measured spin wave frequencies versus the angle of incidence of the light, which, as it was discussed in Sec. 4, is connected with the transferred in-plane wavevector $q_{\|}$. As it is seen in Fig. 11, the obtained dependence deviates from a smooth variation corresponding to Eq. (3). At least two step-like misfits are seen. However, as it was discussed in Sec. 5, confinement of the spin waves and corresponding quantization of the wavevector should cause a splitting in several modes. This was not observed.

Jorzick et al. [26] investigated square lattices of circular permalloy micron dots, similar to those used by Hillebrands et al. [7, 28]. The only difference was the smaller thickness of the dots of $d=40 \mathrm{~nm}$. Fig. 12 shows the anti-Stokes side of a typical BLS spectrum for $q_{\|}=0.42 \cdot 10^{5} \mathrm{~cm}^{-1}$ of an array

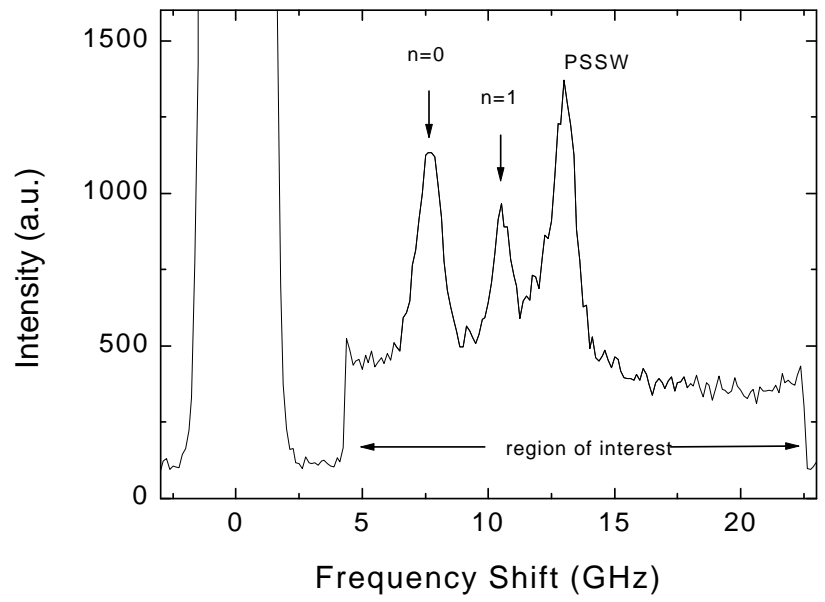

Fig. 12: BLS spectrum of the dot $1 / 1.1 \mu \mathrm{m}$ array with a dot thickness 40 $\mathrm{nm}$, demonstrating the existence of several discrete spin wave modes with a mode splitting of about $2.5 \mathrm{GHz}$. The mode number assignment is indicated. At $13 \mathrm{GHz}$ the lowest order standing spin wave travelling perpendicular to the dot surface (PSSW) is observed as indicated. The applied field is 600 Oe. The transferred in-plane wavevector $q_{\|}$is $0.4 \cdot 10^{5} \mathrm{~cm}^{-1}$. In the region of interest (4-15 GHz) the scan speed was reduced by a factor of three.

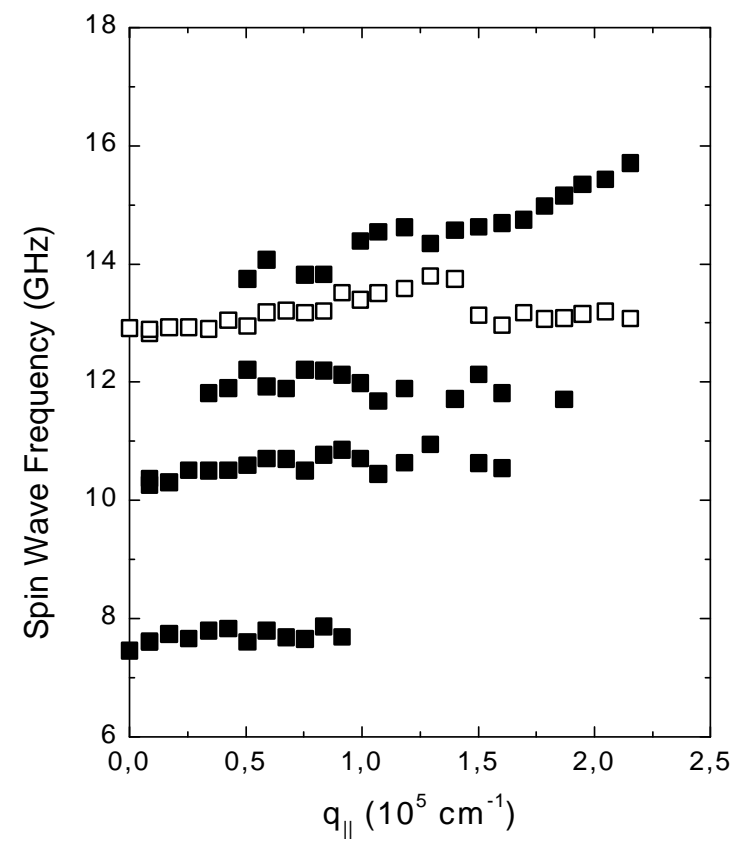

Fig. 13: Obtained dispersion curves for in-plane quantized spin waves (solid squares) and the first perpendicular standing mode (open squares) of the dot $1 / 1.1 \mu \mathrm{m}$ array with a dot thickness $40 \mathrm{~nm}$. The applied field is 600 Oe.

with a dot diameter of $1 \mu \mathrm{m}$ and a dot spacing of $1.1 \mu \mathrm{m}$ at $H=600$ Oe. Similar to the results obtained from the studies of magnetic wires, several peaks, corresponding to in-plane quantized spin wave modes, as well as an exchange dominated, perpendicular standing spin wave mode is seen in Fig. 12. By varying the magnitude of the wavevector, $q_{\|}$, the spin wave dispersion were obtained, as displayed in Fig. 13. Similar to the wire arrays discussed above, each inplane quantized mode is observed within a certain distinct interval of $q_{\|}$, the center of the interval being moved to larger $q_{\|}$with increasing quantization number of the mode compared to the case of wires.

Although the experimental data for the dots look fairly similar to those for the wires, the theoretical analysis of the quantization conditions is presently out of reach, since, as it was mentioned in Sec. 3, a theory of the spin wave quantization in tangentially magnetized dots is not available.

\section{Conclusions}

In the preceding chapters we have seen that BLS is a powerful tool for the investigation of dynamic properties in micron size magnetic islands. Recent BLS experiments have revealed many interesting phenomena such as dynamic size effects, anisotropic magnetic inter-dot coupling, and quantization of spin waves due to in-plane confinement in the islands. Due to a break in lateral translational symmetry the spin wave modes as well as the selection rules in the light scattering process are modified. Despite these successes, some deficiencies, especially in the theoretical description of the spin wave modes in restricted geometries are evident. A consistent further treatment of the problem, where experiment and theory progress together is of great importance. 
References

[1] A. Maeda, M. Kume, T. Ogura, K. Kuroki, T. Yamada, M. Nishikawa, Y. Harada, J. Appl. Phys. 76 (1994) 6667.

[2] R.M.H. New, R.F.W Pease, R.L. White, J. Vac. Sci. Technol. B 12 (1994) 3196.

[3] J.F. Smyth, S. Schultz, D.R. Fredkin, D.P. Kern, S.A. Rishton, H. Schnmid, M. Cali, R.T. Koehler, J. Appl. Phys. 69 (1991) 5262.

[4] N. Bardou, B. Bartenlian, F. Rousseaux, D. Decanini, F. Carcenac, E. Cambril, M.-F. Ravet, C. Chappert, P. Veillet, P. Beauvillain, R. Megy, W. Geerts, J. Ferre, J. Magn. Magn. Mater. 148 (1995) 293.

[5] A.O. Adeyeye, J.A.C. Bland, C. Daboo, J. Lee, U Ebels, H. Ahmed, J. Appl. Phys. 79 (1996) 6120.

[6] M. Grimsditch, Y Jaccard, I.K. Schuller, Phys. Rev. B 58 (1998) 11539.

[7] B. Hillebrands, C. Mathieu, M. Bauer, S.O. Demokritov, B. Bartenlian, C. Chappert, D. Decanini, F. Rousseaux, F. Carcenac: J. Appl. Phys. 81 (1997) 4993.

[8] S.M. Cherif, C. Dugautier, J.-F. Hennequin, P. Moch, J. Magn. Magn. Mater. 175 (1997) 228

[9] A. Ercole, A.O. Adeyeye, J.A.C. Bland, D.G. Hasko, Phys. Rev. B 58 (1998) 345

[10]T. Aign, P. Meyer, S. Lemerle, J. P. Jamet, J. Ferré, V. Mathet, C. Chappert, J. Gierak, C. Vieu, F. Rousseaux, H. Launois, H. Bernas, Phys. Rev. Lett., 81 (1998) 5656.

[11] S.M. Cherif, Y. Roussigne, P. Moch, J.-F. Hennequin, M. Labrune, Proceedings of the 43d Conference on Magnetism \& Magnetic Materials, to be published in J. Appl. Phys..

[12]F. Bloch , Z. Phys. 61 (1930) 206

[13] J.H.E. Griffits, Nature 158 (1946) 670.

[14]P.A. Fleury, S.P.C. Porto, L.E. Cheesman, H.J: Guggenheim, Phys. Rev. Lett. 17 (1966) 84.

[15]C. Kittel, Phys. Rev. 110 (1958) 1295.

[16]L.R. Walker, Phys. Rev. 105 (1956) 390.

[17]R.W. Damon, J.R. Eshbach: J. Phys. Chem. Solids 19 (1961) 308.

[18]P. Grünberg, F. Metawe, Phys. Rev. Lett. 39 (1977) 1561.

[19]R.E. de Wames, T. Wolfram, Appl. Phys. Lett. 16 (1970) 305; J. de Physique 32 (1971) C1-1090.

[20]L.D. Landau, E.M. Lifshitz, Electrodynamics of continuous media, Pergamon Press, Oxford, 1960.

[21]J.R. Sandercock, Trends in Brillouin scattering: Studies of opque materials, supported films, and central modes, in: Light Scattering in Solids III, M. Cardona, G. Güntherodt (eds.), Springer Verlag, Berlin, Heidelberg, New York (1982).

[22]B. Hillebrands, Rev. Sci. Instr., in press (February 1999).

[23] B.A. Gurney, P. Baumgart, V. Speriosu, R. Fontana, A. Patlac, T. Logan, P. Humbert, Digest of the International Conference on Magnetic Films and Surfaces, P7.12, p.474, Glasgow (1991).

[24]C. Mathieu, J. Jorzick, A. Frank, S.O. Demokritov, A.N. Slavin, B. Hillebrands, B. Bartenlian, C. Chappert, D. Decanini, F. Rousseaux, E. Cambrill, Phys. Rev. Lett. 81 (1998) 3968.

[25]B. Hillebrands, S.O. Demokritov, C. Mathieu, S. Riedling, O. Büttner, A. Frank, B. Roos, J. Jorzick, A.N. Slavin, B. Bartenlian, C. Chappert, F. Rousseaux, D. Decanini, E. Cambrill, A. Müller, U. Hartmann, J. Mag. Soc. Jap. 23 (1999) XXX.

[26] J. Jorzick, S.O Demokritov, B. Hillebrands, B. Bartenlian, C. Chappert, F. Rousseaux, to be published.

[27]F. Rousseaux, D. Decanini, F. Carcenac, E. Cambril, M.F. Ravet, C. Chappert, N. Bardou, B. Bartenlian, P. Veillet, J. Vac. Technol. B 13 2787 (1995).

[28]C. Mathieu, C. Hartmann, M. Bauer, O. Büttner, S. Riedling, B. Roos, S.O. Demokritov, B. Hillebrands, B. Bartenlian, C. Chappert, F. Rousseaux, D. Decanini, E. Cambrill, A. Müller, B. Hoffmann, U. Hartmann, Appl. Phys. Lett. 70 (1997) 2912.

[29]B. Hillebrands, Phys. Rev. B 44 (1990) 530. 